\title{
Remarkable structural diversity and single-crystal-to-single-crystal transformations in sulfone functionalized lanthanide MOFs $\dagger$ t
}

\author{
Received 3rd September 2009, Accepted 14th October 2009 \\ First published as an Advance Article on the web 29th October 2009 \\ DOI: $10.1039 / b 918269 c$
}

Eleftheria Neofotistou, ${ }^{a}{ }^{\text {Christos D. Malliakas }}{ }^{b}$ and Pantelis N. Trikalitis ${ }^{* a}$

We report the formation of novel open framework lanthanide (La, Ce, Pr and Dy) MOFs using the ligand 4,4'-bibenzoic acid-2,2'sulfone. In the case of $\mathrm{Ce}$ and $\mathrm{Pr}$, an unprecedented single-crystalto-single-crystal transformation at room temperature was discovered.

Porous, metal-organic frameworks (MOFs) or coordination polymers (PCPs) represent an important class of crystalline open framework solids with diverse chemical composition and pore characteristics $^{1,2}$ and hold great promises for important industrial

${ }^{a}$ Department of Chemistry, University of Crete, Voutes, 71003 Heraklion, Greece. E-mail: ptrikal@chemistry.uoc.gr; Fax: +30 2810 545001; Tel: $+302810545052$

${ }^{b}$ Department of Chemistry, Northwestern University, Sheridan $R d$., Evanston, IL, 60208, USA

$\dagger$ Electronic supplementary information (ESI) available: PXRD, crystallographic data, TGA and additional figures. CCDC reference numbers 736837-736841. For ESI and crystallographic data in CIF or other electronic format see DOI: $10.1039 / \mathrm{b} 918269 \mathrm{c}$

\$ Crystal data for La-2: $\mathrm{C}_{63} \mathrm{H}_{67} \mathrm{~N}_{7} \mathrm{O}_{25} \mathrm{~S}_{3} \mathrm{La}_{2}, M=1696.24$, monoclinic, space group $P 2_{1} / n, a=19.5011(13) \AA, b=16.7853(12) \AA, c=24.2950$ (17) $\mathrm{A}, \beta=112.891(2)^{\circ}, V=7326.2(9) \AA^{3}, T=100(2) \mathrm{K}, Z=4$. Refinement of 901 parameters on 21354 unique reflections out of 161465 measured reflections $\left(R_{\text {int }}=0.0946\right)$ led to $R_{1}=0.0717(I>2 \sigma(I))$, w $R_{2}=0.2192$ (all data). CCDC-736840.

Crystal data for Ce-2: $\mathrm{C}_{60} \mathrm{H}_{60} \mathrm{~N}_{6} \mathrm{O}_{24} \mathrm{~S}_{3} \mathrm{Ce}_{2}, M=1625.56$, monoclinic, space group $P 2{ }_{1} / n, a=19.711(4) \AA, b=18.928(4) \AA, c=23.826(5) \AA$, $\beta=116.74(3)^{\circ}, V=7938(3) \AA^{3}, T=100(2) \mathrm{K}, Z=4$. Refinement of 856 parameters on 11543 unique reflections out of 55316 measured reflections $\left(R_{\text {int }}=0.0565\right)$ led to $R_{1}=0.042(I>2 \sigma(I))$, w $R_{2}=0.0969$ (all data). CCDC-736837. Electron density contributions from disordered guest molecules were handled using the SQUEEZE procedure from the PLATON software suit.

Crystal data for Ce-3: framework formula $\mathrm{C}_{28} \mathrm{H}_{12} \mathrm{O}_{12} \mathrm{~S}_{2} \mathrm{Ce}, M=744.62$, trigonal, space group $R \overline{3}, a=b=25.0566(12) \AA, c=29.944(3) \AA, \alpha=\beta=$ $90^{\circ}, \gamma=120^{\circ}, V=16281.3(19) \mathrm{A}^{3}, T=100(2) \mathrm{K}, Z=9$. Refinement of 195 parameters on 4088 unique reflections out of 52820 measured reflections $\left(R_{\text {int }}=0.0815\right)$ led to $R_{1}=0.079(I>2 \sigma(I)), \mathrm{w} R_{2}=0.2168$ (all data). CCDC-736838. Electron density contributions from disordered guest molecules were handled using the SQUEEZE procedure from the PLATON software suit.

Crystal data for $\operatorname{Pr}-2: \mathrm{C}_{60} \mathrm{H}_{60} \mathrm{~N}_{6} \mathrm{O}_{24} \mathrm{~S}_{3} \mathrm{Pr}_{2}, M=1627.14$, monoclinic, space group $C 2 / c, a=35.0141(19) \AA, b=14.9351(8) \AA, c=$ $30.3411(17) \AA, \beta=97.4220(10)^{\circ}, V=15733.6(15) \AA^{3}, T=100(2) \mathrm{K}, Z=$ 8. Refinement of 856 parameters on 35429 unique reflections out of 206217 measured reflections $\left(R_{\mathrm{int}}=0.0554\right)$ led to $R_{1}=0.0591(I>$ $2 \sigma(I)), \mathrm{w}_{2}=0.1601$ (all data). CCDC-736841.

Crystal data for Dy: $\mathrm{C}_{60} \mathrm{H}_{59} \mathrm{~N}_{6} \mathrm{O}_{25} \mathrm{~S}_{3} \mathrm{Dy}_{2}, M=1685.31$, triclinic, space group $P \overline{1}, a=25.022(4) \AA, b=17.7008(16) \AA, c=22.3869(18) \AA, a=$ $79.657(7)^{\circ}, \beta=80.452(7)^{\circ}, \gamma=81.326(8)^{\circ}, V=4767.6(7) \AA^{3}, T=130(2)$ $\mathrm{K}, Z=2$. Refinement of 865 parameters on 16433 measured reflections $\left(R_{\text {int }}=0.1589\right)$ led to $R_{1}=0.0714(I>2 \sigma(I)), \mathrm{w} R_{2}=0.2047$ (all data). CCDC-736839. Electron density contributions from disordered guest molecules were handled using the SQUEEZE procedure from the PLATON software suit. applications, especially in the field of gas storage ${ }^{3}$ and catalysis. ${ }^{4}$ These multifunctional materials result from molecular self-assembly reactions usually under hydro(solvo)thermal conditions between metal ions or clusters and bridging multidentade organic ligands.

The large number of available organic linkers, offers not only the potential for the construction of structurally and topologically diverse MOFs but also the opportunity to create functionalized porous frameworks with the functional species (atoms or groups) being exposed inside the pore space. This could lead to tailor-made materials for specific applications. ${ }^{5}$ However, while the currently extensive studies are focus on MOFs based on transition metals, lanthanide MOFs are also considered of equal importance., ${ }^{2,6}$

Recently, we published two novel sulfone functionalized $\mathrm{Zn}$-based MOFs (UoC-1 and UoC-2) with unprecedented inorganic and organic secondary building units (SBUs) and overall structures, using for the first time the ligand 4,4'-bibenzoic acid-2, $2^{\prime}$-sulfone $\left(\mathrm{H}_{2} \mathrm{~L}\right.$, see Scheme 1). ${ }^{7}$ In an effort to explore further the potential to synthesize new MOFs with important properties, using this particular ligand, we have extended our work in the lanthanide family of metal cations.

We have discovered a significant structural diversity using different lanthanide cations (La, Ce, Pr and Dy) and more important, in the same $\mathrm{Ln} / \mathrm{H}_{2} \mathrm{~L} / \mathrm{DMF}$ system, very different phases can be isolated, under different reaction conditions.

The reaction between $\mathrm{La}\left(\mathrm{NO}_{3}\right)_{3} \cdot 6 \mathrm{H}_{2} \mathrm{O}(0.332 \mathrm{mmol})$ and $\mathrm{H}_{2} \mathrm{~L}$ ( $0.164 \mathrm{mmol})$ in $10 \mathrm{ml}$ of DMF at $95^{\circ} \mathrm{C}$ for $12 \mathrm{~h}$ afforded a crystalline phase in high yield $(>80 \%)$, denoted here as La-1. However, the quality of the crystals was not sufficient for single-crystal X-ray diffraction measurements. When the supernatant solution from this mixture was kept at room temperature for two weeks, rod-like single crystals of high quality were formed (see ESI), $\dagger$ denoted here as La-2. The system is a new 3D non-interpenetrating MOF that crystallizes in the monoclinic system (space group $P 2_{1} / c$ ) and features one-dimensional channels, as shown in Fig. 1. The inorganic part in La-2 is made of two crystallographically non-equivalent $\mathrm{La}_{2}\left(\mu_{2}-\mathrm{CO}_{2}\right)_{4}\left(\mathrm{CO}_{2}\right)_{4}$ dimers that bridged together and form one-dimensional chains running down the $a$ axis (see Fig. $1 \& 2$ ). In one dimer, the La1-La1 distance is 4.106(2) $\AA$ while in the other is 4.372(1) $\AA$. The La1-La2 distance between two bridged dimers, is 5.841(1) ^. All La atoms in La-2 were found nine coordinated. The two out of the nine

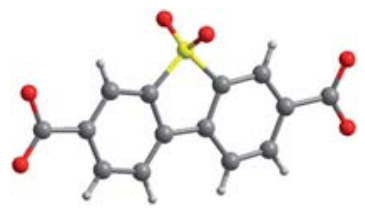

Scheme 1 The non-linear sulfone functionalized ligand $\mathrm{L}^{2-}$. The angle between the terminal carboxylate groups is $163^{\circ}$. 


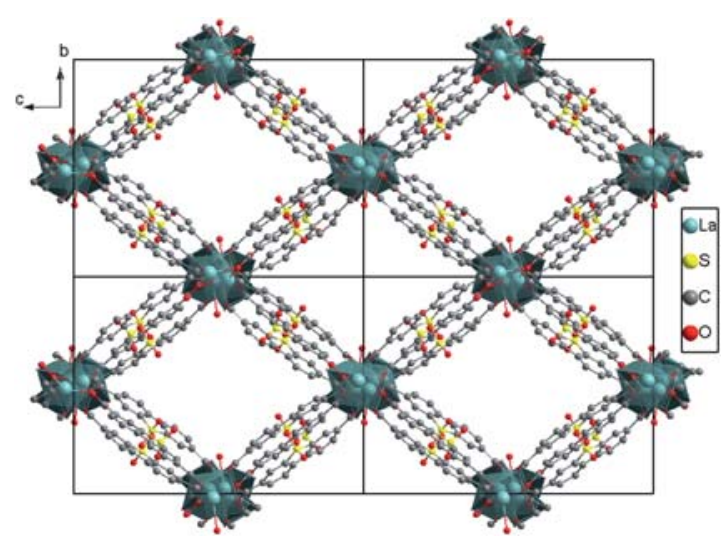

Fig. 1 A $1 \times 1 \times 2$ unit cell representation of La-2 looking down the $a$ axis. Hydrogen atoms and coordinated/guest DMF molecules are omitted for clarity.

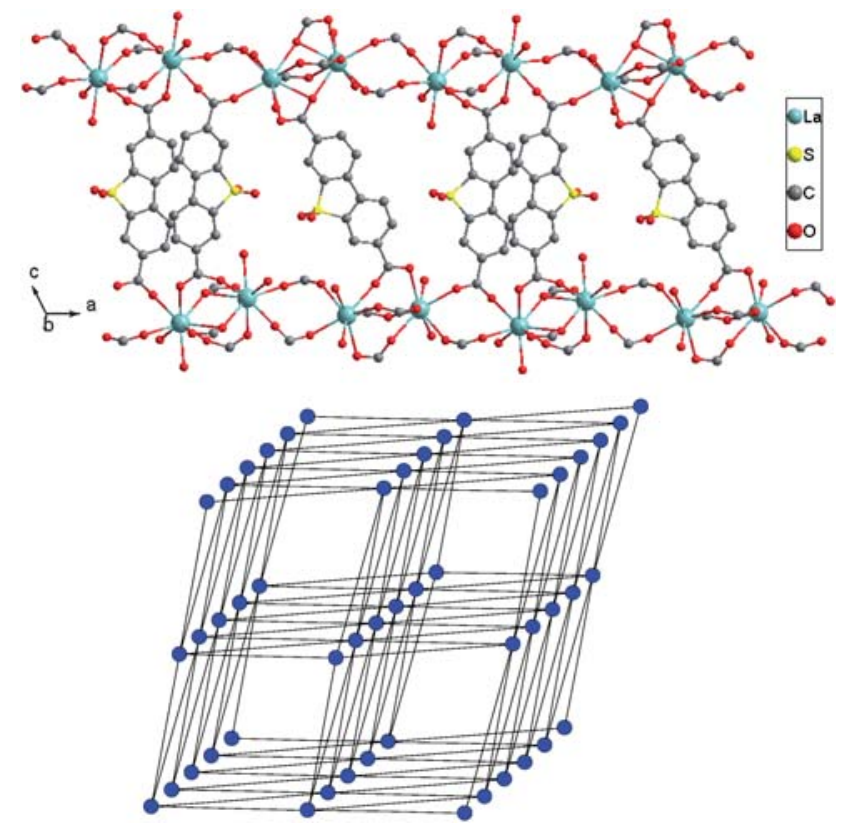

Fig. 2 The connectivity of two adjacent chains in La-2 (top). The dangling oxygen atoms bonded to La atoms represent coordinated DMF molecules. Hydrogen atoms are omitted for clarity. The 8-connected bcu type network topology of La-2 (bottom). Each node of the net (blue circles) represents a $\mathrm{La}-\mathrm{La}$ dimer.

coordination sites are occupied by DMF molecules. Four carboxylate groups serve as a bridge between the two La atoms within each dimer while the four other bridge two La atoms from adjacent dimers. The connectivity between adjacent chains is shown in Fig. 2. Overall, the neutral framework formula is $\mathrm{La}_{2} \mathrm{~L}_{3}(\mathrm{DMF})_{4}$. It is important to note here that the calculated powder X-ray diffraction pattern (PXRD) of La-2 do not match with the experimental PXRD pattern of La-1 (see Fig. S8 in ESI). $\uparrow$ Therefore, La-1 and La-2 are different crystalline phases.

In order to simplify the 3D structure of La-2 and identify the corresponding net topology, we considered the dimers as the central nodes of the net (the center of gravity of each unit is considered as the node) and the organic ligands as connectors. The topological analysis using the software package TOPOS, ${ }^{8}$ revealed that La-2 is an 8-connected bcu type network (Schäfli symbol $4^{24} 6^{4}$ ), as shown schematically in Fig. 2 at the bottom.

In the case of $\mathrm{Ce}$, following an identical synthetic procedure, we first isolated the solid product, Ce-1, from the solvothermal reaction in DMF and the supernatant solution was kept at room temperature. From the later, we isolated the isostructural analogue of La-2, denote here as $\mathrm{Ce}-2$. The $\mathrm{Ce} 1-\mathrm{Ce} 1$ and $\mathrm{Ce} 2-\mathrm{Ce} 2$ distance, of the two crystallographically independent dimers is 4.4643(9) $\AA$ and 4.2568(8) $\AA$, respectively. The $\mathrm{Ce} 1-\mathrm{Ce} 2$ distance between two bridged dimers is 5.6772(13) A (see ESI for single crystal data). $\dagger$

Remarkably, and in contrast to the La-1 phase, we found that the initially isolated solid, Ce-1, consisting of low quality plate-like single crystals, when putted in fresh DMF and kept at room temperature for one month, was quantitatively transformed into high quality large, cubic-like single crystals (see Fig. 3, left). This new phase, denoted here as Ce-3, crystallized in the trigonal system (space group $R \overline{3})$ and is a novel non-interpenetrating $3 \mathrm{D}$ MOF with 3D intersecting channels (see Fig. 3, right). As shown in Fig. 4, the inorganic $\mathrm{SBU}$ is a novel, linear anionic trinuclear cluster, with formula $\left[\mathrm{Ce}_{3}\left(\mathrm{CO}_{2}\right)_{12}\right]^{3-}$. Bond valence calculations are in full agreement with $\mathrm{Ce}^{3+}$. In this $\mathrm{SBU}$, there are two crystallographically non-equivalent $\mathrm{Ce}^{3+}$ atoms and the distance between Ce1-Ce2 is 3.981(4) $\mathrm{A}$. In terms of the coordination environment of $\mathrm{Ce}^{3+}$ atoms in $\mathrm{Ce}-3$ as compared to $\mathrm{Ce}-2$, there are distinct differences; in $\mathrm{Ce}-3$ there are no coordinated DMF molecules and also, while $\mathrm{Ce} 1$ is nine coordinated, $\mathrm{Ce} 2$ is twelve. The $\mathrm{Ce} 1-\mathrm{O}$ and $\mathrm{Ce} 2-\mathrm{O}$ bond lengths are found in the range 2.482(4)-2.556(4) ^ and 2.624(4)-2.699(4), respectively. Notably, as in the case of UoC-1 and UoC-2, ${ }^{7}$ each SBU is linked to six other by twelve $\mathrm{L}^{2-}$ ligands, following a pseudo-octahedral connectivity, as shown in Fig. 4. Accordingly, in each direction, two ligands stack in parallel one on the top of the other and serve as linkers between two adjacent SBUs. The distance between these two ligands is approximately $3.6 \AA$, which is typical of aromatic, face-to-face $\pi-\pi$ stacking. Each pair of ligands is in fact an organic SBU, stabilized by weak $\pi-\pi$ interactions, which link together the six-connected inorganic SBUs (see Fig. 4, right), resulting in the non-interpenetrating 3D open structure of Ce-3. Considering the trimeric units as nodes and the organic SBUs (pairs of $\mathrm{L}^{2-}$ ligands) as single connectors, the network topology of Ce-3 is identical to that of 6-connected single pcu net (Schäfli symbol $4^{12} 6^{3}$ ), as shown schematically in Fig. 4 at the bottom.
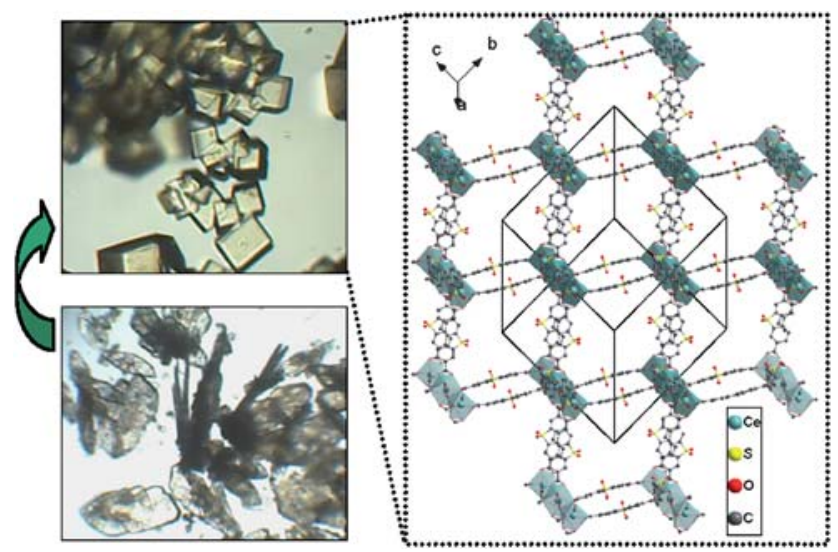

Fig. 3 Optical images of Ce-1 (left, bottom) and Ce-3 (left, top) and a representative unit cell view of Ce-3 (right). The arrow at left indicates the single-crystal-to-single-crystal transformation (see text). 


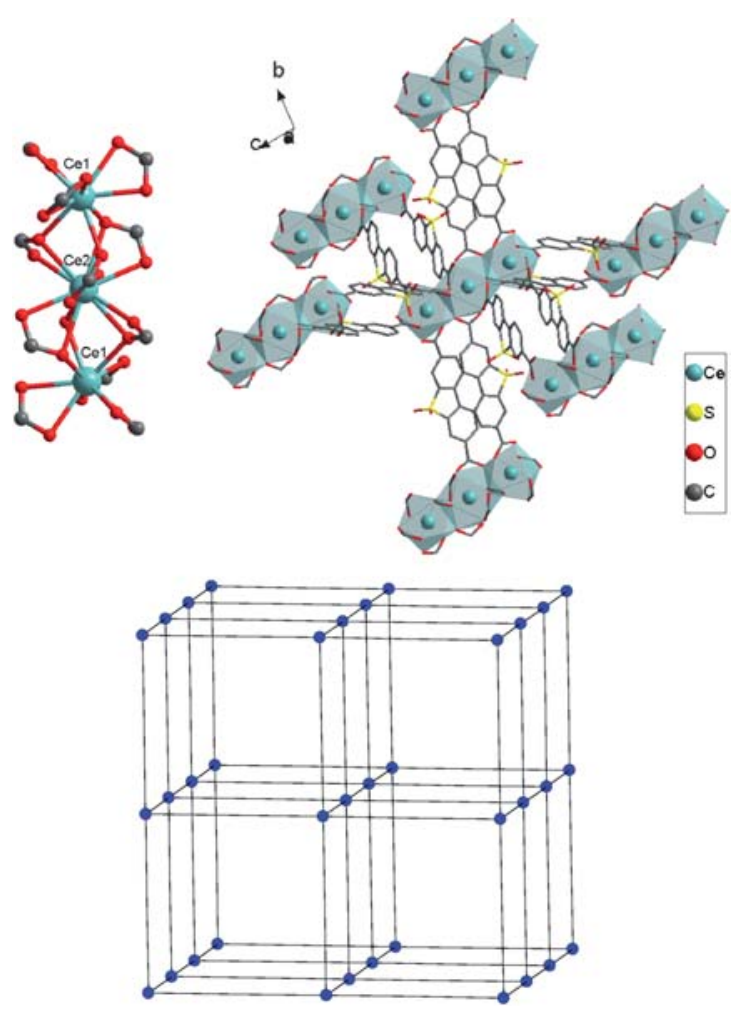

Fig. 4 The novel anionic trinuclear $\left[\mathrm{Ce}_{3}\left(\mathrm{CO}_{2}\right)_{12}\right]^{3-} \mathrm{SBU}$ (top left) in Ce-3 and its pseudo-octahedral connectivity to six adjacent SBUs, through six pairs of $\mathrm{L}^{2-}$ ligands (top right). The 6-connected pcu type network topology of Ce-3 (bottom). Each node of the net (blue circles) represents a cerium trimer.

The single-crystal X-ray diffraction analysis of Ce-3 did not reveal an exact location of charge balancing cations for the anionic framework $\left[\mathrm{Ce}_{3} \mathrm{~L}_{6}\right]^{3-}$. Given the fact that it is generally difficult to completely formulate the exact composition of all guest molecules in highly porous MOFs and also that dimethylammonium cations, $\left(\mathrm{CH}_{3}\right)_{2} \mathrm{NH}_{2}{ }^{+}$, are formed in situ upon heating of DMF, ${ }^{9}$ it is natural to consider these as charge balancing cations. In combination with thermogravimetric analysis (TGA) results (see Fig. S3 in ESI), $\dagger$ the overall chemical formula of Ce-3 is $\left[\left(\mathrm{CH}_{3}\right)_{2} \mathrm{NH}_{2}\right]_{3}\left[\mathrm{Ce}_{3} \mathrm{~L}_{6}\right](\mathrm{DMF})_{6}$.

In the case of Pr, based on the PXRD pattern of the solid isolated after the solvothermal reaction in DMF, Pr-1 (again, poor quality crystals, see Fig. S4 in ESI), $\dagger$ we may conclude that this phase is isostructural to Ce-1 (see Fig. S9 in ESI). $\dagger$ When this solid was transferred in a fresh DMF solution and kept at room temperature for a period of one month, it was quantitatively transformed into the isostructural $\mathrm{Ce}-3$ phase, again through a remarkable single-crystalto-single-crystal transformation (see Fig. S4 in ESI). $\dagger$ Accordingly, this phase is denoted here as $\operatorname{Pr}-3$. Despite this notable similarity with the Ce system, the supernatant solution from the Pr reaction did not yield the isostructural $\mathrm{Ce}-2$ and $\mathrm{La}-2$. In contrast, the isolated phase denoted here as Pr-2, is a 2-fold interpenetrating 3D MOF made of simple $\operatorname{Pr}$ dimers linked together through a single $\mathrm{L}^{2-}$ ligand, following a pseudo-octahedral connectivity (Fig. S6 and S7 in ESI). $\dagger$ The Pr1-Pr2 distance is 4.2803(2) $\AA$. This material is isostructural with the very recently reported $\mathrm{Nd}, \mathrm{Eu}$ and $\mathrm{Gd}$ phases obtained with the ligand $\mathrm{H}_{2} \mathrm{~L} .{ }^{10}$ The topological analysis ${ }^{8}$ revealed that these $3 \mathrm{D}$ MOFs are examples of 2-fold interpenetration related by a center of symmetry (Class IIa) ${ }^{11}$ with an sxb network topology (Schäfli symbol $\left.4^{8} 5^{4} 6^{3}\right) .{ }^{10}$

In the case of Dy, the solvothermal reaction afforded good quality single crystals, suitable for X-ray structural determination. No other phases were obtained in this system. This material is a new 3D noninterpenetrating MOF crystallized in the triclinic system (space group $P \overline{1}$ ) and features 3D intersecting channels (see Fig. 5). The framework is made of isolated $\mathrm{Dy}_{2}\left(\mu_{2}-\mathrm{CO}_{2}\right)_{4}\left(\mathrm{CO}_{2}\right)_{2}$ dimers, each of which is linked to six other by six $\mathrm{L}^{2-}$ ligands; four of them adopt a bis(bidentate) coordination mode while the other two adopt a bis(chelating) mode (see Fig. 5 right). Interestingly, there are two crystallographically non-equivalent dimers; the Dy1-Dy1 and Dy2Dy2 distance is 4.1027(10) $\AA$ and 4.2444(13) $\AA$, respectively. All Dy atoms are found eight coordinated, having two of the coordination sites being occupied by DMF molecules. The overall neutral framework formula is $\mathrm{Dy}_{2} \mathrm{~L}_{3}(\mathrm{DMF})_{4}$. Considering the dimers as nodes and the $\mathrm{L}^{2-}$ ligands as connectors, the network topology of Dy is 6connected single pcu (Schäfli symbol $4^{12} 6^{3}$ ), similar to that shown in Fig. 4 at the bottom.

The structural diversity discovered in the lanthanide series $\mathrm{La}, \mathrm{Ce}$, Pr and Dy could be explained in part by the well known lanthanide contraction phenomenon. Accordingly, in the isostructural neutral framework phases La-2 and Ce-2, the large size of the nine coordinated lanthanide atoms results in bridged dimers while in the case of Pr-2, the smaller size of the nine coordinated Pr atoms results in isolated dimers and two-fold interpenetration. The situation does not change in moving to $\mathrm{Nd}, \mathrm{Eu}$ and $\mathrm{Gd}$ as the corresponding phases are isostructural with $\mathrm{Pr}-2$. However, in going to Dy, interpenetration is not any more allowed due the smaller eight coordinated Dy atoms. It is important to note here that the above crystalline phases based on the $\mathrm{H}_{2} \mathrm{~L}$ ligand are different that those reported in some lanthanides using the non-functionalized parent ligand 4,4-biphenyl dicarboxylic acid. ${ }^{12}$ The origin of this structural diversity is attributed in part to both the non-linear bridging mode of the ligand $\mathrm{H}_{2} \mathrm{~L}$ (the angle between the two carboxylate end-groups is $163^{\circ}$ ) and its increased acidity caused by the electronegative sulfone group attached to the phenyl rings.

An unprecedented discovery in this work is the room temperature single-crystal-to-single-crystal quantitative transformation of yet unknown Ce-1 and Pr-1 to the remarkable 3D open-framework, isostructural Ce-3 and $\mathrm{Pr}-3$, respectively. It is important to note here that no product was formed when the corresponding lanthanide salts were combined with $\mathrm{H}_{2} \mathrm{~L}$ ligand in DMF solution and kept at room temperature for months. These results suggest that the initial solvothermal reaction is very crucial, promoting not only the necessary deprotonation of $\mathrm{H}_{2} \mathrm{~L}$ molecules and presumably the formation of lanthanide building blocks, but also the decarbonylation of DMF
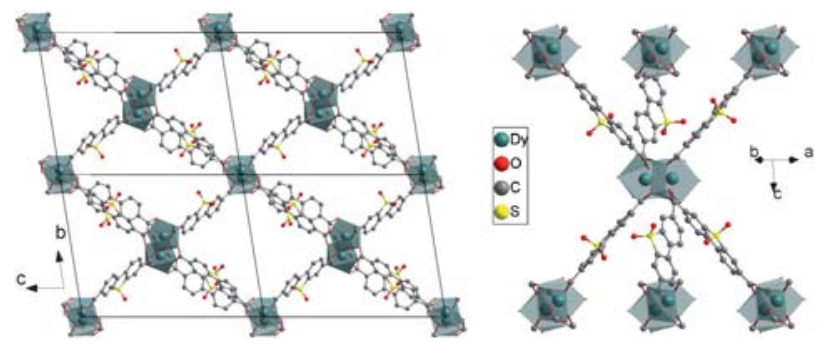

Fig. 5 A $2 \times 2 \times 1$ representation of Dy looking down the $a$ axis (left) and the corresponding six-connected dimeric SBUs (right). 
and the release of dimethylamine. The later can easily protonated and act as a counterion for the construction of $\mathrm{Ce}-3$ and Pr-3. After this important step, the interplay between thermodynamic and kinetic parameters presumably controls the formation of the final structure, in each particular system. We are currently exploring different reaction conditions in the above systems in order to understand and elucidate the formation mechanism of the different phases.

Finally, we currently investigate the properties of these materials and in particular, the possibility to remove the guest molecules from the open-framework solids La-2, Ce-2, Ce-3, Pr-3 and Dy and access the available pore space.

\section{Acknowledgements}

Financial support by EU (75\%) and the Greek Government (25\%) through the program PENED-2003 code numbers 03ED581 and 03ED450, Interreg IIIA Greece-Cyprus (K2301.004) and European Commission DG RTD (NESSHY, Contract SES6-518271) is gratefully acknowledged. ChemMatCARS Sector 15 is principally supported by NSF/Department of Energy (DOE) under grant number CHE-0535644. The Advanced Photon Source at Argonne National Laboratory is supported by the DOE, Office of Science, Office of Basic Energy Sciences, contract No. DE-AC02-06CH11357. We are grateful to Prof. Mercouri G. Kanatzidis for providing access to single-crystal XRD equipment. We also thank Prof. Giannis $\mathrm{S}$. Papaefstathiou for assisting in topological analyses.

\section{References}

1 (a) S. Kitagawa and R. Matsuda, Coord. Chem. Rev., 2007, 251, 24902509; (b) O. M. Yaghi, M. O'Keeffe, N. W. Ockwig, H. K. Chae, M. Eddaoudi and J. Kim, Nature, 2003, 423, 705-714; (c) A. K. Cheetham, C. N. R. Rao and R. K. Feller, Chem. Commun., 2006, 4780-4795; (d) S. J. Dalgarno, N. P. Power and J. L. Atwood, Coord. Chem. Rev., 2008, 252, 825-841; (e) P. Jin, S. J. Dalgarno, C. Barnes, S. J. Teat and J. L. Atwood, J. Am. Chem. Soc., 2008, 130, 17262.

2 G. Ferey, Chem. Soc. Rev., 2008, 37, 191-214.

3 (a) A. W. C. van den Berg and C. O. Arean, Chem. Commun., 2008, 668-681; (b) J. L. C. Rowsell and O. M. Yaghi, Angew. Chem., Int.
Ed., 2005, 44, 4670-4679; (c) M. Dinca and J. R. Long, Angew. Chem., Int. Ed., 2008, 47, 6766-6779; (d) R. Banerjee, A. Phan, B. Wang, C. Knobler, H. Furukawa, M. O'Keeffe and O. M. Yaghi, Science, 2008, 319, 939-943; (e) Y. S. Bae, K. L. Mulfort, H. Frost, P. Ryan, S. Punnathanam, L. J. Broadbelt, J. T. Hupp and R. Q. Snurr, Langmuir, 2008, 24, 8592-8598; (f) K. S. Iyer, M. Norret, S. J. Dalgarno, J. L. Atwood and C. L. Raston, Angew. Chem., Int. Ed., 2008, 47, 6362-6366.

4 M. Heitbaum, F. Glorius and I. Escher, Angew. Chem., Int. Ed., 2006, 45, 4732-4762; S. Horike, M. Dinca, K. Tamaki and J. R. Long, J. Am. Chem. Soc., 2008, 130, 5854.

5 (a) K. K. Tanabe, Z. Wang and S. M. Cohen, J. Am. Chem. Soc., 2008, 130, 8508-8517; (b) S. Hasegawa, S. Horike, R. Matsuda, S. Furukawa, K. Mochizuki, Y. Kinoshita and S. Kitagawa, J. Am. Chem. Soc., 2007, 129, 2607-2614; (c) A. D. Burrows, C. Frost, M. F. Mahon and C. Richardson, Angew. Chem., Int. Ed., 2008, 47, 8482-8486.

6 (a) R. E. Morris and P. S. Wheatley, Angew. Chem., Int. Ed., 2008, 47, 4966-4981; (b) L. Pan, K. M. Adams, H. E. Hernandez, X. T. Wang, C. Zheng, Y. Hattori and K. Kaneko, J. Am. Chem. Soc., 2003, 125, 3062-3067; (c) S. Kitagawa, R. Kitaura and S. Noro, Angew. Chem., Int. Ed., 2004, 43, 2334-2375; (d) T. K. Maji, G. Mostafa, H. C. Chang and S. Kitagawa, Chem. Commun., 2005, 2436-2438; (e) Y. Q. Sun, J. Zhang, Y. M. Chen and G. Y. Yang, Angew. Chem., Int. Ed., 2005, 44, 5814-5817; $(f)$ T. Devic, O. David, M. Valls, J. Marrot, F. Couty and G. Ferey, J. Am. Chem. Soc., 2007, 129, 12614.

7 E. Neofotistou, C. D. Malliakas and P. N. Trikalitis, Chem.-Eur. J., 2009, 15, 4523-4527.

8 V. A. Blatov and A. P. Shevchenko, TOPOS 4.0, Samara State University, Russia, 2008.

9 A. D. Burrows, K. Cassar, R. M. W. Friend, M. F. Mahon, S. P. Rigby and J. E. Warren, CrystEngComm, 2005, 7, 548-550.

10 L. Yan, Q. Yue, Q.-X. Jia, G. Lemercier and E.-Q. Gao, Cryst. Growth Des., 2009, 9, 2984-2987.

11 (a) I. A. Baburin, V. A. Blatov, L. Carlucci, G. Ciani and D. M. Proserpio, J. Solid State Chem., 2005, 178, 2452-2474; (b) I. A. Baburin, V. A. Blatov, L. Carlucci, G. Ciani and D. M. Proserpio, Cryst. Growth Des., 2008, 8, 519-539; (c) V. A. Blatov, L. Carlucci, G. Ciani and D. M. Proserpio, CrystEngComm, 2004, 6, 378-395.

12 (a) X. D. Guo, G. S. Zhu, Q. R. Fang, M. Xue, G. Tian, J. Y. Sun, X. T. Li and S. L. Qiu, Inorg. Chem., 2005, 44, 3850-3855; (b) Y. F. Han, X. H. Zhou, Y. X. Zheng, Z. Shen, Y. Song and X. Z. You, CrystEngComm, 2008, 10, 1237-1242. 